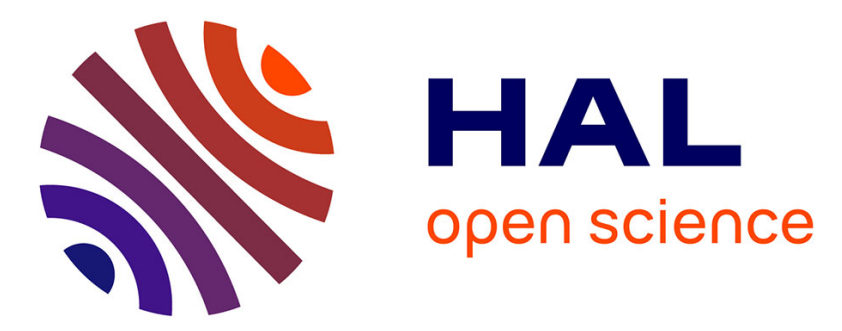

\title{
Propagation of capillary waves and ejection of small droplets in rapid droplet spreading
}

Hui Ding, E.Q. Li, F.H. Zhang, Yi Sui, Peter D.M. Spelt, S. T. Thoroddsen

\section{To cite this version:}

Hui Ding, E.Q. Li, F.H. Zhang, Yi Sui, Peter D.M. Spelt, et al.. Propagation of capillary waves and ejection of small droplets in rapid droplet spreading. Journal of Fluid Mechanics, 2012, 697, pp.92-114. hal-00744484

\section{HAL Id: hal-00744484 \\ https://hal.science/hal-00744484}

Submitted on 23 Oct 2012

HAL is a multi-disciplinary open access archive for the deposit and dissemination of scientific research documents, whether they are published or not. The documents may come from teaching and research institutions in France or abroad, or from public or private research centers.
L'archive ouverte pluridisciplinaire HAL, est destinée au dépôt et à la diffusion de documents scientifiques de niveau recherche, publiés ou non, émanant des établissements d'enseignement et de recherche français ou étrangers, des laboratoires publics ou privés. 


\title{
Propagation of capillary waves and ejection of small droplets in rapid droplet spreading
}

\author{
H. Ding ${ }^{1,2}$, E. Q. Li $^{3,4}$, F. H. Zhang ${ }^{3,5}$, Y. Sui ${ }^{6}$, P. D. M. Spelt ${ }^{6,7} \dagger$ \\ and S. T. Thoroddsen ${ }^{4}$ \\ ${ }^{1}$ Department of Modern Mechanics, University of Science and Technology of China, \\ 230027 Hefei, China \\ 2 Department of Chemical Engineering, University of California at Santa Barbara, \\ Santa Barbara, CA 93106-5080, USA \\ ${ }^{3}$ Department of Mechanical Engineering, National University of Singapore, \\ 119260 Singapore \\ ${ }^{4}$ Division of Physical Sciences and Engineering \& CCRC, King Abdullah University of Science and \\ Technology, Thuwal, 23955-6900, Saudi Arabia \\ ${ }^{5}$ Singapore-MIT Alliance, National University of Singapore, 117576 Singapore \\ ${ }^{6}$ Department of Chemical Engineering, Imperial College London, London SW7 2AZ, UK \\ ${ }^{7}$ Département Mécanique, Université de Lyon 1, and Laboratoire de la Mécanique des Fluides \& \\ d'Acoustique, CNRS, 69134 Ecully, France
}

(Received 15 September 2010; revised 14 November 2011; accepted 23 January 2012; first published online 12 March 2012)

\begin{abstract}
A new regime of droplet ejection following the slow deposition of drops onto a near-complete wetting solid substrate is identified in experiments and direct numerical simulations; a coalescence cascade subsequent to pinch-off is also observed for the first time. Results of numerical simulations indicate that the propagation of capillary waves that lead to pinch-off is closely related to the self-similar behaviour observed in the inviscid recoil of droplets, and that motions of the crests and troughs of capillary waves along the interface do not depend on the wettability and surface tension (or Ohnesorge number). The simulations also show that a self-similar theory for universal pinch-off can be used for the time evolution of the pinching neck. However, although good agreement is also found with the double-cone shape of the pinching neck for droplet ejection in drop deposition on a pool of the same liquid, substantial deviations are observed in such a comparison for droplet ejection in rapid drop spreading (including the newly identified regime). This deviation is shown to result from interference by the solid substrate, a rapid downwards acceleration of the top of the drop surface and the rapid spreading process. The experiments also confirm non-monotonic spreading behaviour observed previously only in numerical simulations, and suggest substantial inertial effects on the relation between an apparent contact angle and the dimensionless contact-line speed.
\end{abstract}

Key words: drops, capillary waves, contact lines 


\section{Introduction}

Drop impact on a dry surface can result in a range of fascinating physical phenomena such as rebound, splashing and crown formation (cf. Yarin 2006). Although slow deposition of drops onto a substrate normally may be expected to lead to the mere gradual spreading of drops, several studies on complete or near-complete wetting cases have reported inertial effects (Biance, Clanet \& Queré 2004; Ding \& Spelt 2007a) and, for sufficiently rapid spreading, part of the drop was found to be ejected (Roux \& Cooper-White 2004; Rioboo et al. 2006). A typical time sequence for the ejection of a small droplet, referred to as a daughter droplet herein, is shown in figure 1. A similar series of events has been reported by Thoroddsen \& Takehara (2000), Blanchette \& Bigioni (2006) and Gilet et al. (2007) in the partial coalescence of drops with a pool of the same liquid; wave focusing leads eventually to pinch-off and not to a large diverging pressure wave as, for instance, in the collapse of a bubble or cavity (Shaw \& Spelt 2010) or whip cracking (Goriely \& McMillen 2002), although a small sound wave has been measured in, for instance, the snap-off of a bubble (Manasseh, Riboux \& Risso 2008). The degree to which self-similar capillary-wave propagation (e.g. Keller \& Miksis 1983) and a pinching regime (e.g. Eggers 1997; Day, Hinch \& Lister 1998) are approached in these systems has not yet been analysed, however. Furthermore, reducing empiricism in direct numerical simulations of flows with moving contact lines remains a challenge and the flows studied herein provide an opportunity to see to what extent the numerical results compare well with experimental values. The objective of this study is therefore threefold: to report a new mechanism of droplet ejection in drop spreading, to analyse capillary-wave propagation and pinchoff using self-similar theory and finally to compare the numerical and experimental results. We make use of the following dimensionless groups: Ohnesorge number $O h=\mu / \sqrt{\rho \sigma R_{0}}$ and Weber number $W e=\rho V^{2} R_{0} / \sigma$, where $R_{0}, \rho$ and $\mu$ are the radius, density and viscosity of the drop, respectively; $\sigma$ is the surface tension and $V$ is the impact speed. The surface hydrophilicity is characterized by the static contact angle. Experiments show that the ejection of daughter droplets generally occurs in the slow deposition of drops of low viscosity, therefore, all of the cases considered herein have relatively small values of $\mathrm{Oh}$ and $W e$.

We refer to the regime shown in figure 1 as first-stage pinch-off. In this regime, the rapid spreading on the substrate stretches the drop horizontally, which generates a capillary-type wave that travels up from the contact line region towards and focuses on the top of the drop, leading initially to a column-like shape and eventually to a pinching neck at the bottom of the column. In this process the contact-line motion effectively acts as a wave maker. Apart from aiming to classify when such flow regime (as well as another, previously unidentified regime) occurs, we also analyse the propagation of the capillary wave up along the droplet. Capillary waves have of course been studied in detail previously, including standing waves on freely oscillating spherical droplets (Rayleigh 1879). Renardy et al. (2003) demonstrated the existence of pyramidal structures that arise from the speed of a travelling capillary wave being annulled by the impact velocity if the latter is sufficiently large, and a scaling argument was used to model this shape. Thoroddsen et al. (2007b) studied capillary-Marangoni waves using a self-similar description, for a drop coalescing with a flat pool of a different liquid. The pioneering work of Keller \& Miksis (1983) has identified self-similar far-field solution of propagating waves on a slender wedge. Subsequent work in this area has been on slender wedges involving a moving contact line (King 1991), and the recoil of fat cones (Billingham 1999). In $\S 3.2$, we analyse 


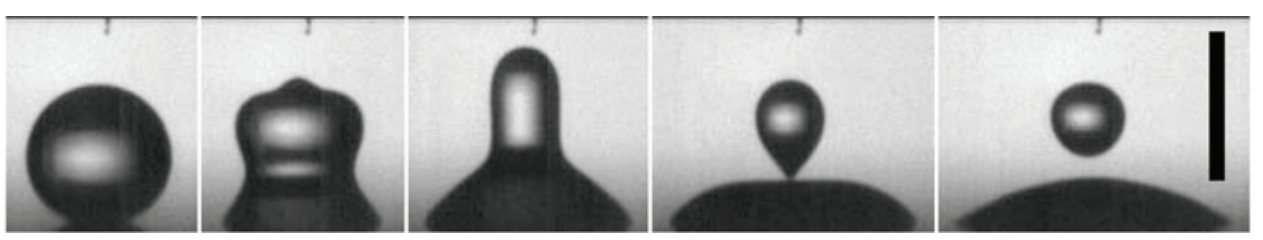

FIGURE 1. First-stage pinch-off for a water drop of $0.486 \mathrm{~mm}$ in diameter $(O h=0.0069)$ deposited on a hydrophilic glass substrate $\left(\theta_{s}=12^{\circ}\right)$, at times $0,0.28,0.56,0.86$ and $1.82 \mathrm{~ms}$. The scale bar is $0.5 \mathrm{~mm}$.

the capillary wave on an undisturbed surface that is approximately spherical and driven by the moving contact line at low-impact velocities.

Furthermore, we determine whether a universal neck pinching regime is approached at late times. Specifically, the double-cone structure of Day et al. (1998) in a potentialflow regime is expected to be relevant here. A transition to an inertial-viscous thread is only expected (as can be inferred from e.g. Lister \& Stone 1999) when the radius of the pinching neck (made dimensionless with $R_{0}$ ), which is at most $10^{-4}$ in this study, is of $O h^{2}$. This is very late and only just before breakup, so the potential-flow regime is expected to dominate most of the pinch-off process. In the systems studied here, mainly water drops in air are studied, so a further transition to a Stokes flow regime is not expected to be observed before molecular scales are approached (Lister \& Stone 1999). As yet, it is unclear to what extent the potential-flow regime can be recovered in the present system, or whether the presence of the solid substrate delays such an approach. These issues are investigated in $\S \S 3.1$ and 3.3 .

Previous work has also identified inertial regimes in droplet spreading, which appear to deviate from those in classical analyses of e.g. Cox (1986), although ejection of small droplets was not reported. Ding \& Spelt (2007a) showed that in rapid spreading without pinch-off, after a capillary-type wave travels from the contact line towards the top of the droplet, the droplet rapidly collapses, leading to a large, radially outward motion inside the drop. A boundary layer created by this flow may interfere with the structure of the contact-line region, and the results of Ding \& Spelt (2007a) revealed that an apparent contact angle ceases to be a single-valued function of the contact-line speed. We therefore investigate in the experiments in $\S 3.4$ whether this behaviour is also observed.

We close with an investigation in the effect of the size of the inner contact-line region and a discussion of the criteria for pinch-off in $\S 3.5$, and observations of a coalescence cascade (wherein a sequence of droplets of decreasing size are produced) subsequent to the first-stage pinch-off in $§ 3.6$.

\section{Methodology}

\subsection{Experimental set-up}

Our experiments indicate that even minute impact velocities have an adverse effect on the occurrence of droplet ejection. Therefore, we attempt herein to deposit droplets onto a solid surface with as small an impact velocity as possible $(W e<0.05)$. We use gravity-driven pinch-off from minuscule needles, made in a glass puller, of diameter in the range $5-100 \mu \mathrm{m}$. The tip of one needle is visible in figure 1 . Direct pinch-off can generate $650 \mu \mathrm{m}$ diameter droplets and by softly tapping the nozzle we can produce droplets as small as $450 \mu \mathrm{m}$. We also use distilled water, and in some cases acetone, methanol, ethanol and glycerin mixtures to adjust the wettability of the liquid on 
the solid surface and the $O h$ number through changing the viscosity. The substrate was microscopic glass slides, which were washed with acetone, dried and subjected to oxygen plasma treatment $(0.4$ Torr, $150 \mathrm{~W})$ for $2 \mathrm{~min}$ in a Triple P Plasma Processor (Duratek). The root-mean-square surface roughness of the untreated surface was $\sim 2 \mathrm{~nm}$, while after the oxygen plasma treatment, this reduced to around $1 \mathrm{~nm}$, see Jang et al. (2010). This treatment generated surfaces with various hydrophilicities. Since the hydrophilicity was not perfectly uniform over the entire surface of the same plate and reduced over time, the apparent contact angles were measured within about 70 ms following the overall dynamic phase (which lasted for few milliseconds), with an error of about $\sim 4^{\circ}$. This criterion of the measure of the angles ensured that no significant variation of these by either the overall dynamic phase or the phase changes near the contact line (which became significant in time of seconds).

For each droplet deposition, a part of the substrate was used that had not been used previously, hence there was no liquid film present prior to droplet deposition. The dynamics are observed with either a Photron SA-1 or SA-5 CMOS video cameras with frame rates up to 100000 frames $\mathrm{s}^{-1}$.

\subsection{Numerical method}

In order to gain further insight into the flow behaviour we also use a diffuse interface method (Ding, Spelt \& Shu 2007) for simulations of pinch-off processes, which is capable of simulating two-phase flows with large density and viscosity ratios and accounts for moving contact lines (Ding \& Spelt 2008; Ding, Gilani \& Spelt 2010). The wettability is prescribed by an advancing contact angle $\theta_{A}$ and a hysteresis window $\Delta \theta$. Note that when the drop finally rests on the solid substrate after impact, its static contact angle could be an arbitrary value within $\left(\theta_{A}-\Delta \theta, \theta_{A}\right)$. The fluid-fluid interface is presented by the volume fraction of one fluid $C$, and tracked by solving the convective Cahn-Hilliard equation:

$$
\frac{\partial C}{\partial t}+\nabla \cdot(\boldsymbol{u} C)=(1 / P e) \nabla^{2} \psi,
$$

where $\psi\left(=C n^{-1} \phi^{\prime}(C)-C n \nabla^{2} C\right)$ is the chemical potential, $\phi\left(=C^{2}(1-C)^{2} / 4\right)$ is the bulk energy density, $C n\left(=\epsilon / R_{0}\right)$ is the Cahn number and $\epsilon$ represents a measure of the thickness of the diffuse interface; $P e=M_{c} \phi_{c} / V$ is the Péclet number, $M_{c}$ and $\psi_{c}$ are the characteristic values of the mobility and the chemical potential, respectively. In this study, we choose $\mathrm{Pe}=\mathrm{Cn}^{-2}$, which ensures that the diffusive fluxes on the right-hand side of (2.1) go to zero when $C n$ approaches zero (Jacqmin 1999). Similar to other interface capturing methods (such as level-set and volume-of-fluid methods), changes in topology, such as pinch-off or coalescence, occur automatically in the diffuse interface method when the distance between interfaces becomes of the order of the grid spacing (here related to the thickness of the interfacial region). At the solid substrate, the diffuse interface model uses a no-slip boundary condition for velocity, and a geometric formulation of wetting conditions provides a hydrodynamic boundary condition for volume fraction $C$ (Ding \& Spelt 2007b). The stress singularity at moving contact line is alleviated by the diffusive fluxes in the contact line region, of which the magnitude is controlled by a constant mobility and Cn (Jacqmin 2000). The governing equations are solved on a uniform MAC grid; further details can be found in Ding et al. (2007). Unless stated otherwise, a structured mesh of $800 \times 800$ has been used for a domain of $3.6 \times 3.6$.

In Ding \& Spelt (2007a), test results were found to compare favourably with those obtained with a level-set method wherein a slip condition was used, if the slip 
length $\lambda$ was chosen to be proportional to $C n$ (when using a constant mobility). The value of $C n$ cannot be arbitrarily small and is restricted by the grid size. We choose $C n=0.0025$ and the resultant effective thickness of the diffuse interface (i.e. the distance between $C=0.1$ and 0.9 ) is $\sim 0.01$. For a millimetre-size drop, this suggests the corresponding slip length is $\sim 2.5 \mu \mathrm{m}$, which falls short of realistic conditions, e.g. $\lambda \sim O(0.1 \mu \mathrm{m})$ at most (Marsh, Garoff \& Dussan 1993). As a larger effective slip length tends to give rise to faster droplet spreading, this would lead to a quantitative difference between numerical results and experiments despite the use of otherwise identical flow parameters such as surface tension, contact angles, viscosities and drop size, etc. Therefore, in our simulations we slightly adjust the experimental values, such as $\theta_{A}$ and/or $O h$, to reproduce the flow phenomena observed in experiments, and on this basis analyse the related flow mechanisms that are not readily amenable to experimental analysis. A hybrid method incorporating PARAMESH (MacNeice et al. 2000), an open-source adaptive mesh refinement (AMR) tool for parallel computing, has been developed by us recently, which has enabled us to also perform highresolution simulations with a dimensionless effective slip length down to $O\left(10^{-4}\right)$ (which is computationally very expensive, even with AMR). Extensive tests and detailed comparisons with theoretical results of this method will be reported elsewhere; here, it is used to supplement the results obtained without AMR to study in $\S 3.5$ the role of effective slip length, facilitating a quantitative comparison with experiment, and in $\S 3.6$ the coalescence cascade.

\section{Results}

Unless stated otherwise, the results have been made dimensionless with the inertialcapillary time $T=\left(\rho R_{0}^{3} / \sigma\right)^{1 / 2}$, and the initial drop radius $R_{0} ; t=0$ represents the time when the drop comes into contact with the solid substrate.

\subsection{Second-stage pinch-off: overall flow behaviour}

Our experiments and simulations have revealed a previously unidentified regime of droplet ejection by rapidly spreading drops, which we shall refer to as second-stage pinch-off. Figure $2(a, b)$ shows the series of events that result in second-stage pinch-off. The simulations also predict this behaviour (figure $2 c$ ), albeit for somewhat different settings, for reasons explained in $\S 2.2$ above (this is discussed further in $\S 3.5$ ). The second-stage pinch-off process results in the ejection of a small droplet, of which the size ranges from 0.1 to 0.2 in experiments and $0.20-0.23$ in simulations. The subsequent behaviour in figure $2(b)$ suggests impact of the daughter droplet on the main drop on the substrate, and then a subsequent bounce-off. This event has not been identified previously in spreading processes, although an at first sight a similar pinchoff process has been reported in the studies of pinch-off resulting from coalescence of non-equal-sized droplets (Blanchette \& Bigioni 2009; Zhang, Li \& Thoroddsen 2009). Figure 3 shows the parameter space of pinch-offs obtained by experiments. Second-stage pinch-off regime is observed between first-stage pinch-off and no-pinchoff, and occurs at $O h$ in the range of $0.004-0.01$. The regime transition shows a strong dependency on the contact angle: in general, first no-pinch-off, followed by secondstage pinch-off and then first-stage pinch-off is expected when gradually decreasing the contact angle. Deposition of a droplet that is very small does not lead to a small daughter droplet pinching off, because the value of $O h$ would be too large. Droplets that are sufficiently large are expected to show second-stage pinch-off of a daughter 
(a)

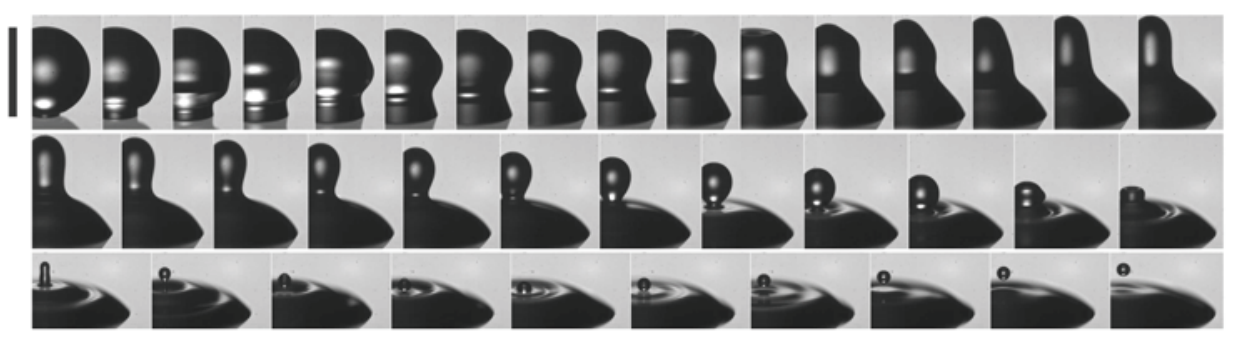

(b)

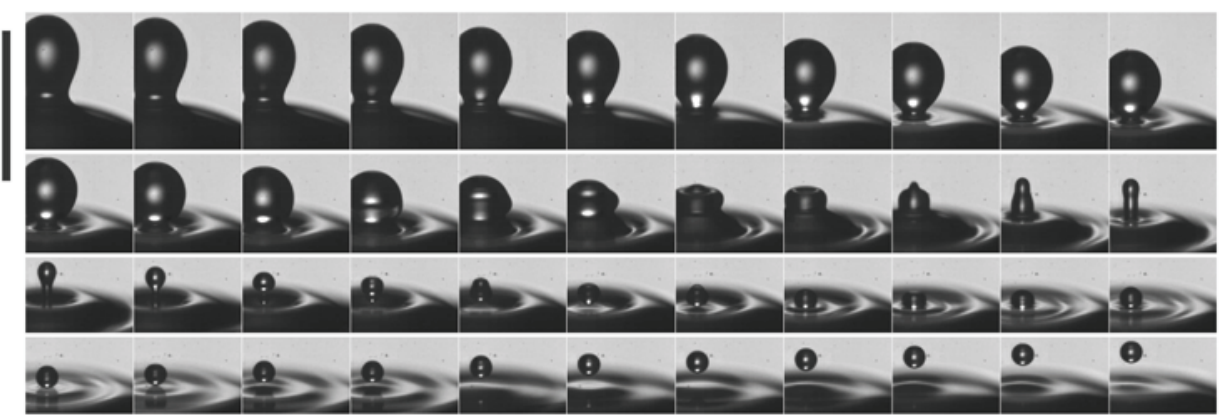

(c)

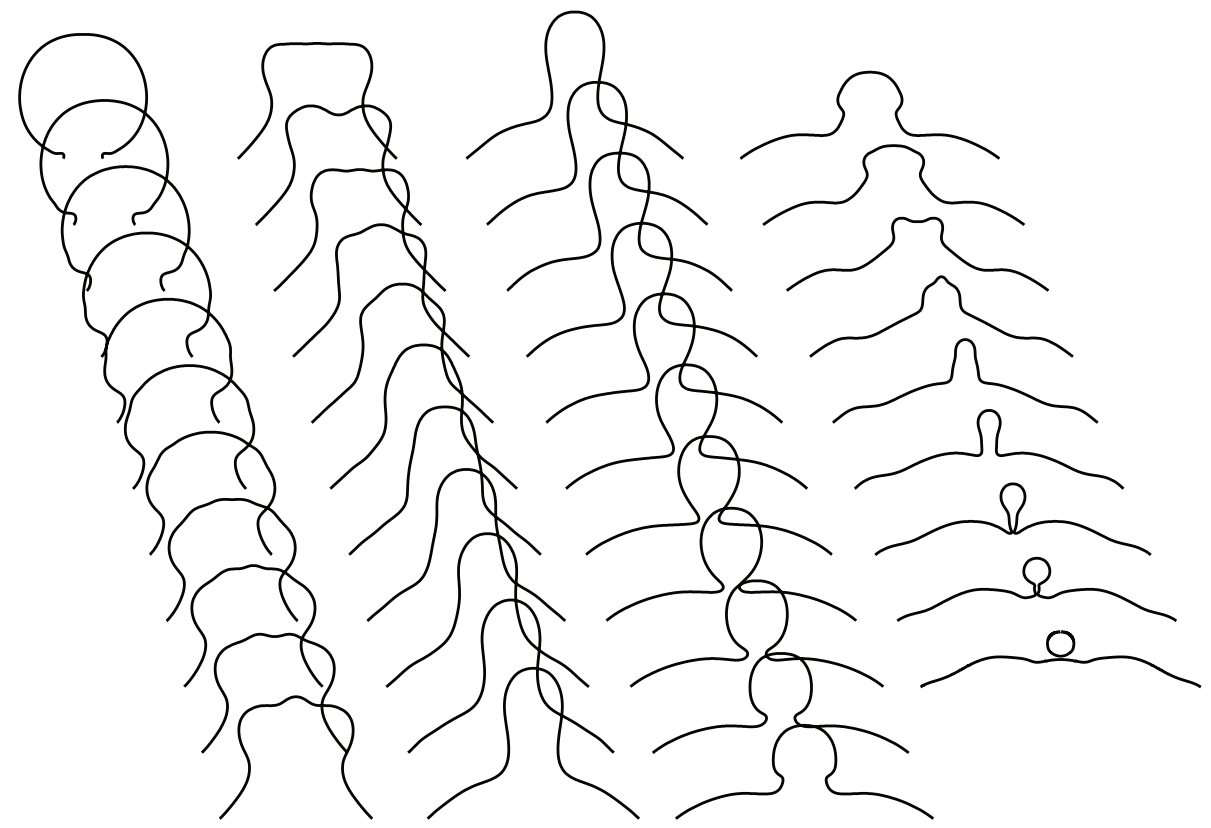

FIGURE 2. Typical shape evolution of a second-stage pinch-off. (a) Overall pinch-off process for a drop of water with frames spaced by $100 \mu \mathrm{s}$. The droplet diameter is $1.016 \mathrm{~mm}$, the impact speed is $0.037 \mathrm{~m} \mathrm{~s}^{-1}(O h=0.0047$ and $W e=0.019)$ and $\theta_{s}=22.2^{\circ}$; video recorded by setting the camera obliquely at $14^{\circ}$, and the scale bar is $1 \mathrm{~mm}$. (b) Close-up of the second stage, with frames spaced by $40 \mu \mathrm{s}$, the scale bar is $1 \mathrm{~mm}$, see the accompanying supplementary movie 1 (available at http://dx.doi.org/10.1017/jfm.2012.49). (c) Numerical results for $O h=0.0066, W e=0.011, \theta_{A}=35^{\circ}$ and $\Delta \theta=10^{\circ}$ with time running from top to bottom, and then from left to right. 


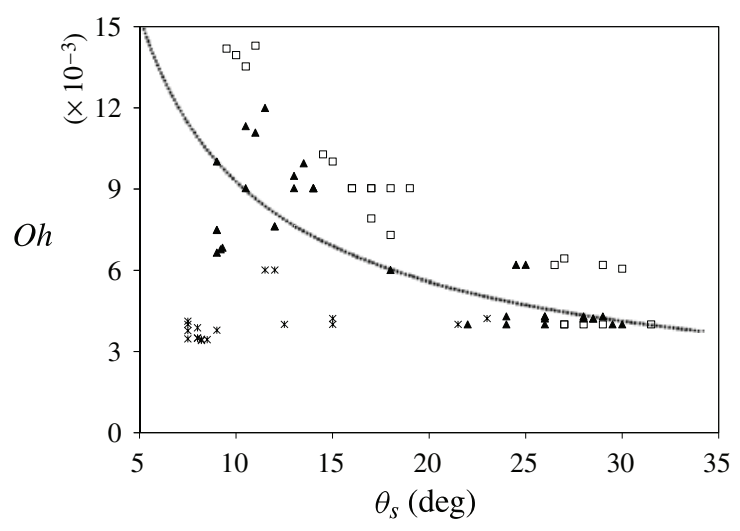

FIGURE 3. Parameter space of pinch-offs obtained by experiments. Triangles show secondstage pinch-off in the region between no pinch-off $(\square)$ and first-stage pinch-off $(*)$. The dotted line is drawn to guide reader's eye.

droplet or, for even larger droplets, first-stage pinch-off. The results shown in figure 3 are discussed further in $\S 3.5$ below.

As is evident from the experimental observations using an oblique view (figure $2 a, b$ ), first-stage pinch-off almost occurs in second-stage pinch-off (the last frame in the first row of figure $2 b$ ), but the pinching neck re-expands at a late stage. Second-stage pinch-off eventually occurs between the second and third frame in the third row of figure $2(b)$. Even if the side view of the neck in the experiments of second-stage pinch-off is obscured by a ridge on the drop surface (as is the case in the measurement of contact-line speed and apparent contact angle), one can still distinguish between second-stage pinch-off and first-stage pinch-off with subsequent coalescence and ejection (see, for example, the accompanying supplementary movie 2) through careful observation of capillary waves in the neck region, which would be expected as a result of any pinch-off. We have not observed such waves after the first time a droplet tries to pinch off in the accompanying supplementary movie 1.

\subsection{Capillary waves}

Capillary waves result from the difference between the initial apparent and the static contact angle. When a drop is gently deposited onto the wall, the interface, locally making an angle with the wall that corresponds to the contact angle, must bend over itself further upwards to an angle of nearly $180^{\circ}$, due to the (near-spherical) shape of the original drop. As a result, high curvature is generated near the contact line, i.e. an initial disturbance to interface that subsequently propagates up the droplet. Therefore, the smaller the contact angle, the larger the local curvature and the stronger the wave.

Travelling capillary waves have been studied extensively following the work of Keller \& Miksis (1983) on surface-tension-driven flows in a wedge, including the extension to cones for the inviscid recoil of droplets (e.g. Lawrie 1990; Billingham 1999; Sierou \& Lister 2004), to slender cones with a contact line (King 1991) and to a drop coalescing with a flat pool of a different liquid (Thoroddsen et al. 2007b). The spatial propagation of the capillary wave appears to render a comparison with the theory of Rayleigh (1879) for the amplification of standing waves on a sphere or cylinder not useful. Although the geometry studied in these analyses differs, 


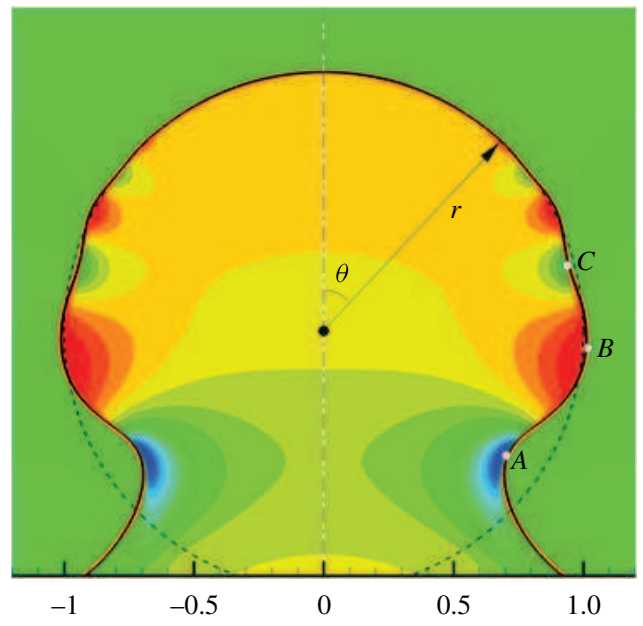

FIGURE 4. (Colour online available at journals.cambridge.org/flm) Measurement of capillary waves in a polar coordinate $(\theta, r)$, where a sphere of original drop size (dashed curve) is anchored at the drop peak. Such measurement is made until the waves reach the drop peak, i.e. $\theta=0^{\circ}$. The colour contours represent the pressure field. Propagation of the primary capillary wave, marked by points $A, B$ and $C$, will be investigated later in this section.

especially the near field, we investigate briefly whether at least the (self-similar) far-field determined in these studies is relevant here.

The far-field determined first in Keller \& Miksis (1983) for slender wedges, by Lawrie (1990) for slender cones and by Billingham (1999) for fat cones, is in selfsimilar coordinates $(R, \xi)$ of the form

$$
R=\alpha \xi^{-q} \sin \left(\frac{4}{27} \xi^{3}-\beta\right)
$$

In order to define $R$ and $\xi$, we first introduce a polar system $(\theta, r)$ that is anchored at the top of the drop (figure 4), which moves downwards with increasing time. In Billingham (1999), $\xi$ is the radial coordinate and in effect the radial axis virtually coincides with the undisturbed interface; $R$ is an effective height of the interfacial disturbance. Therefore, in the present context, $R$ is taken as the value of $r$ at the interface in dimensionless form, $R=(r-1) t^{-2 / 3} ; \xi$ is the corresponding coordinate along the undisturbed interface, which is here the arclength $\left(\theta_{0}-\theta\right) t^{-2 / 3}$ and $\theta_{0}$ is the value of $\theta$ when the drop comes into contact with the solid substrate (note that the radius of the undisturbed sphere is unity); $\alpha$ and $\beta$ are constants. For slender wedges and cones, $q=7 / 2$ (Keller \& Miksis 1983; Lawrie 1990; King 1991); for fat cones, $q=5$ (Billingham 1999).

A typical result of wave propagation is shown in figure 5(a) for a drop at relatively low $O h$, where at least three periods of the wave are seen to travel from the initial contact line $(\theta \approx \pi)$ towards the peak of the drop $(\theta=0)$; also, see accompanying supplementary movie 3 for more details. Note that although the drop is initially spherical in all simulations, it is slightly deformed by the time it approaches the solid wall. This results in a non-uniform $r$ distribution seen at early times in figure 5(a).

In figure $5(b)$, the interface shape as obtained from the numerical simulations is shown in the self-similarity coordinates $(R, \xi)$. We conclude from this result that a self-similar shape is not exactly attained, although the differences between the curves is not large. An exact self-similar solution should not be expected here, due to the 

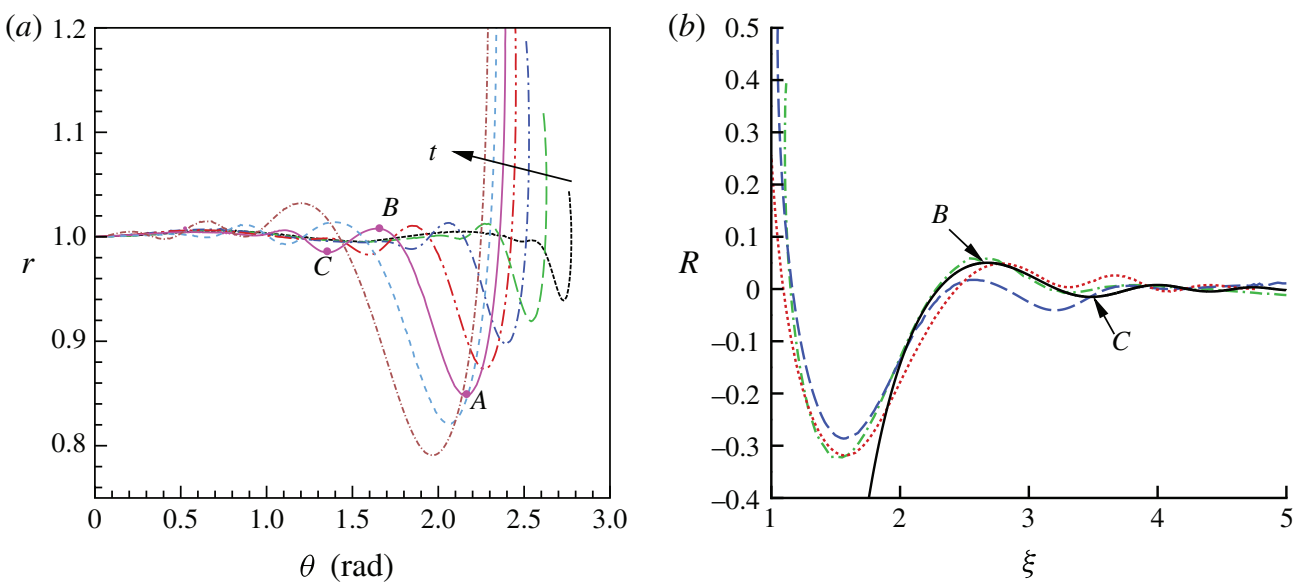

Figure 5. (Colour online) Capillary wave propagation. (a) A typical wave, propagating from right to left at $O h=0.008, W e=0.016, \theta_{A}=30^{\circ}$; time intervals of 0.073 . (b) Comparison with asymptotic self-similar theory. Solid line is the far field of Billingham (1999), broken lines are numerical results: dash-dotted line, $t=0.11$, dashed line, 0.29 , dotted line, 0.47 .

significant differences between the simulations and the ideal conditions in the analysis. For example, compared with the analysis by Billingham (1999), here the angle that the undisturbed cone makes with the substrate varies much more strongly, the origin of the polar system moves towards the solid wall (due to the impact velocity), the wavelength is not very short with respect to the drop radius and the path of the wave is rather finite. Nevertheless, the comparison of this shape with (3.1) with fitted values of the amplitude $\alpha(=8)$ and phase $\beta(=1.8)$ seems not bad; although we have used the result of Billingham (1999), the result for thin wedges gives little difference on the scale of the uncertainty observed. Note that (3.1) is most relevant to the far field, and a near-field correction is required for it to approach the contact line. Finally, we observe that the wave at early times (e.g. $t=0.29$ ) in figure $5(b)$ appears to have been damped somewhat, whereas the wave at relatively late times (e.g. $t=0.47$ ) seems stronger than the others. A possible reason for this is that in the former, most of the wave is below the equator, whereas most of the wave is above the equator in the latter (see figure $5 a$ ). Convergence amplification leads to pinch-off, as shown clearly for bubble coalescence in Zhang \& Thoroddsen (2008).

More detailed analysis substantially strengthens the quantitative comparison between the numerical results and theoretical analysis (3.1). In figure 6, the amplitude and angular location of the points $B$ and $C$ in figure 4 are compared with the corresponding predictions from (3.1). The theoretical predictions shown here are determined as follows: in figure $5(b)$ points $B$ and $C$ are seen to be located on the self-similar solution at $\xi=2.65$ and 3.5 , respectively, therefore, their $R$ values follow by using these values in (3.1); then the prediction of time-dependent amplitude and angular location of $B$ and $C$ can be made by recalling $r=1+R t^{2 / 3}$ and $\theta_{0}-\theta=\xi t^{2 / 3}$. Given that the initial drop shape was not exactly spherical at impact, and that the capillary wave reaches the top of the drop at $t=0.5$, the comparison of the amplitude is reasonable, and the location of the points $B$ and $C$ is well represented by (3.1).

The wave propagation for cases of first-stage and second-stage pinch-off (obtained by varying wettability or $O h$ ) are also shown in figures 6 and 7. The angular locations 

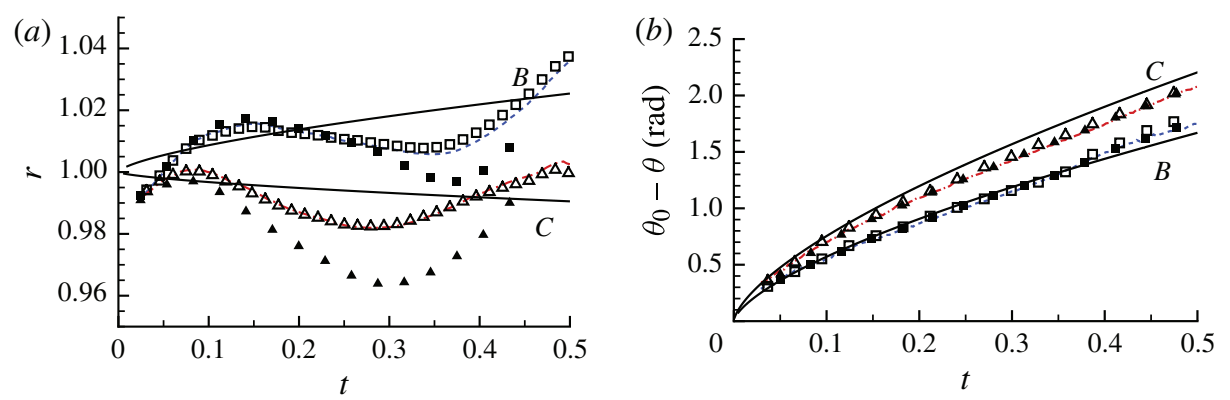

FIgURE 6. (Colour online) The dimensionless wave amplitude $(a)$ and angular location $(b)$ of points B (dashed line and squares) and $\mathrm{C}$ (dash-dotted line and triangles) indicated in figures 4 and 5. The solid lines are the predictions from (3.1). The dashed and dash-dotted lines are the results for $O h=0.008$ and $\theta_{A}=30^{\circ}$ (second-stage pinch-off), the empty symbols for $O h=0.008$ and $\theta_{A}=15^{\circ}$ (first-stage pinch-off), and the filled symbols for $O h=0.004$ and $\theta_{A}=30^{\circ}$ (first-stage pinch-off). Note, the capillary wave reaches the top of the drop around $t=0.5$.

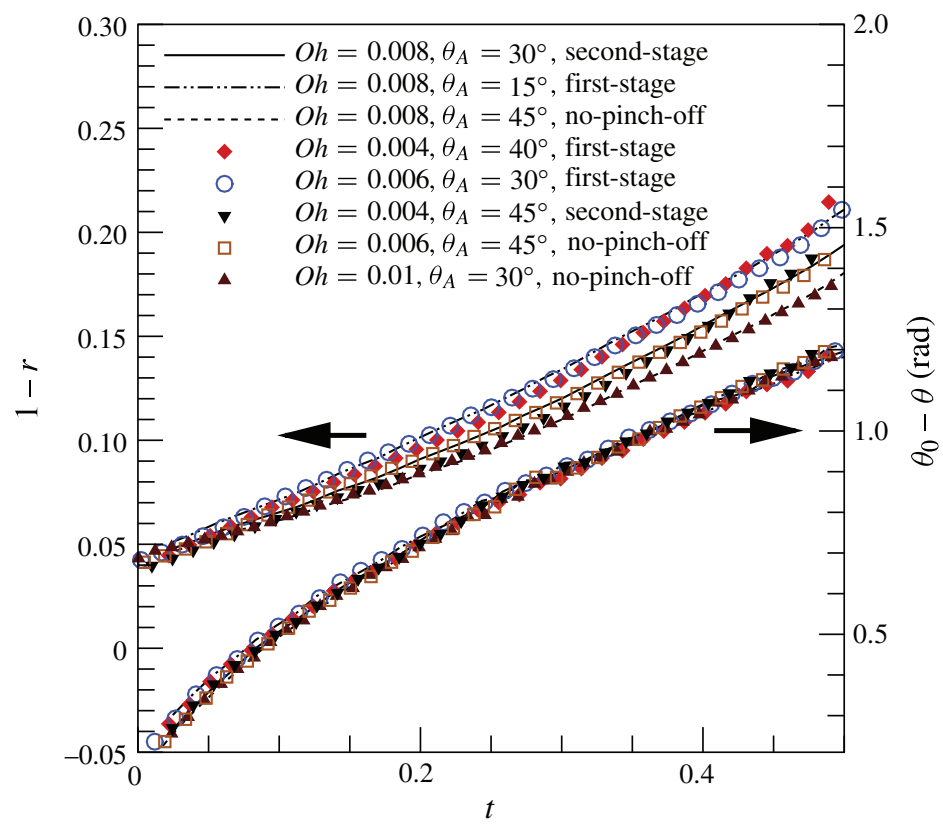

FIgURE 7. (Colour online) The dimensionless wave amplitude and angular location of point $A$ as a function of time for different pinch-off regimes.

of the waves (in terms of $A, B$ and $C$ ) can be seen to be very similar in time for all cases, whereas the amplitudes are not, especially at point $A$. A retrospective study of figure 2 shows that points $B$ and $C$ propagate towards the drop peak and eventually damp out there, while point $A$, the trough closest to the moving contact line, persists and evolves into a neck at a later time. Therefore, it is expected that a useful way to characterize the flow regimes is through the propagation of point $A$. It is confirmed in figure 7, in which the amplitude at point $A$ as a function of time appears to be different for the various pinch-off regimes. This is true for most of 

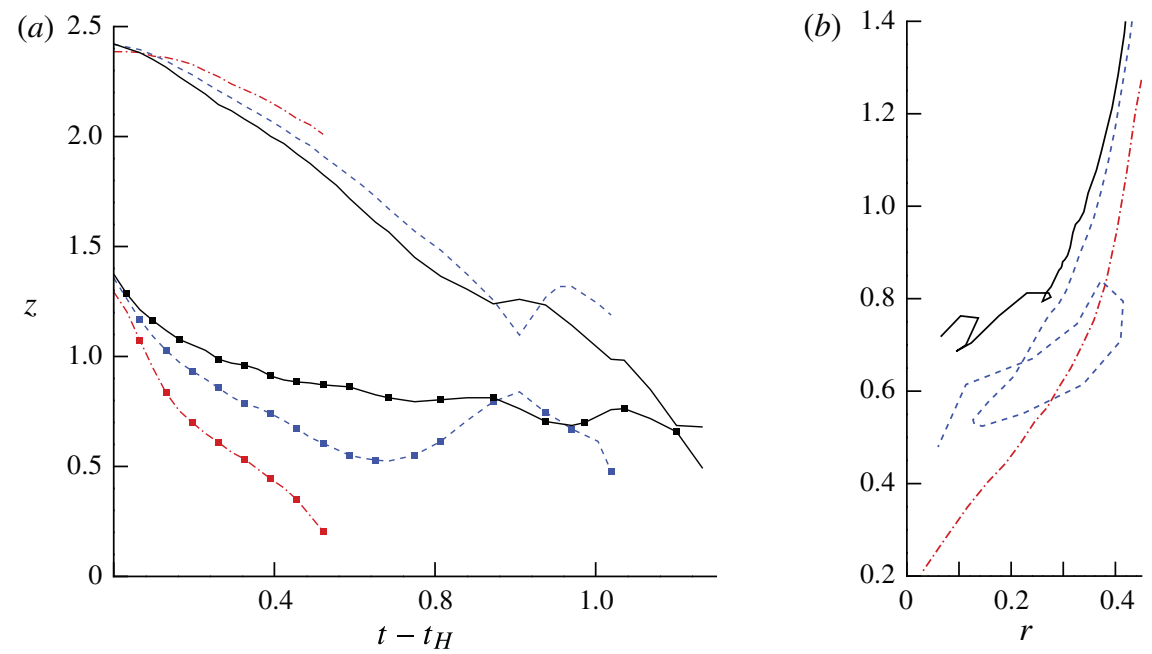

FIGURE 8. (Colour online) Simulation results for dynamic pinching neck at $W e=0.011$ and $O h=0.0066$ : $(a)$ the drop height (solid lines without symbols) and $z$-location of the pinching neck (lines with symbols) as a function of time from the time $t_{H}$ when the top of the drop reaches it highest position; $(b)$ the location of the pinching neck in the $(r, z)$ plane, for first-stage pinch-off (red dash-dotted lines, $\theta_{A}=15^{\circ}$ ), second-stage pinch-off (blue dashed lines, $\theta_{A}=40^{\circ}$ ) and no pinch-off (black solid lines, $\theta_{A}=45^{\circ}$ ).

the studied cases, except for $O h=0.006$ and $\theta_{A}=45^{\circ}$, which finally evolves into no pinch-off although the amplitude growth of $A$ suggests a second-stage pinch-off. The evolution of droplet shape of this case, however, shows that it is in fact very close to a second-stage pinch-off case. Figure 7 also shows that second-stage pinch-off lies in between first-stage pinch-off and no pinch-off with respect to $\theta_{A}$ or $O h$, in agreement with experiments (see figure 3). The propagation of point $A$ at later times, when it leads to a pinching neck, is studied in more detail in the next subsection.

\subsection{Pinch-off}

The simulations provide further insight into the approach to pinch-off. Figure $8(a)$ shows that in the case which eventually leads to second-stage pinch-off, the drop height initially decreases faster than the $z$-location of the pinching neck. As may be anticipated, and as confirmed by figure 2 and the accompanying supplementary movie 1 , this increases the cone angle and therefore interferes with the pinching process: such bulging-out of the upper cone intensifies the competition between principle curvatures at the neck, making the axial curvature sharper than the azimuthal curvature and resulting in expansion of the neck. Figure 9 displays two typical second-stage pinchoff patterns that occur in our simulations. For the first type, as shown in figure $9(a)$, the bulge of the top cone is beginning to show at a late time of the pinching process and proceeds until pinch-off. The second type is more dramatic as seen in figures $2(b)$ and $9(b)$ : the top cone does not bulge but gradually evolves into an ice-cream-conelike shape, with two pinching necks. On a regime map of $\theta_{A}$ versus $O h$, the first type would be close to no-pinch-off while the second type would be close to first-stage pinch-off.

The minimum radius of the pinching neck, $R_{\min }$ (measured from the symmetry axis) is shown in figure 10, as a function of time to rupture $t_{R}-t$, where $t_{R}$ is the rupture 

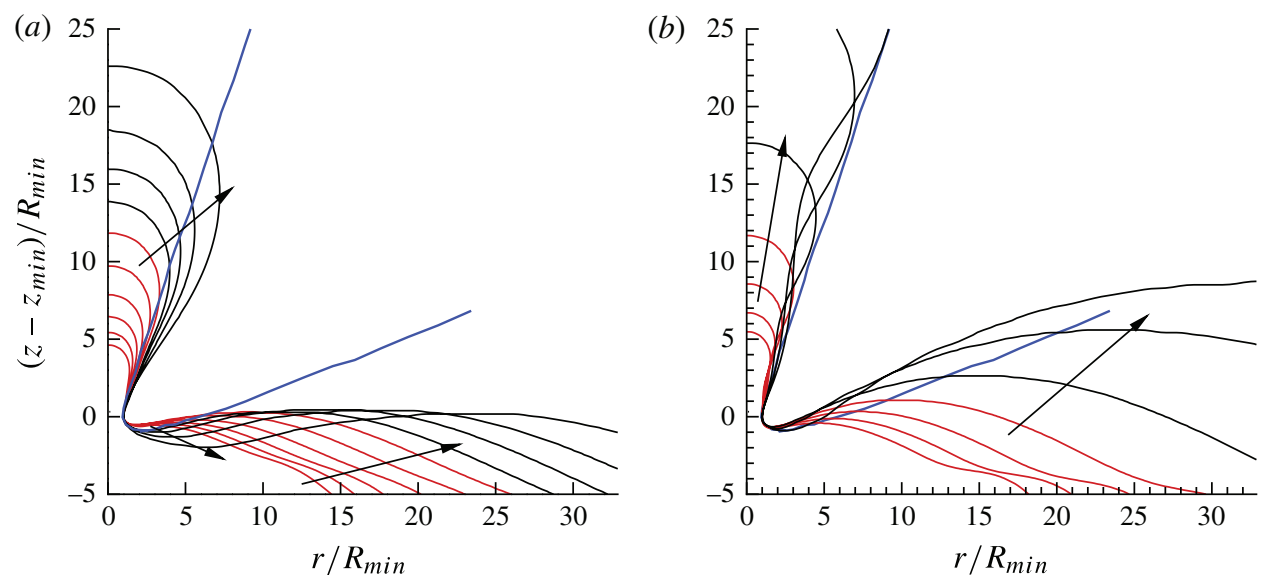

Figure 9. (Colour online) Comparison of scaled interface shapes for two second-stage pinch-off patterns at $W e=0.011$ and $O h=0.0066$ : $(a) \theta_{A}=40^{\circ}$ and $\Delta \theta=10^{\circ}$; and $(b)$ $\theta_{A}=35^{\circ}$ and $\Delta \theta=10^{\circ}$. The shapes highlighted in red show the $\left(t_{R}-t\right)^{2 / 3}$ regime for $0.08<t_{R}-t<0.2$. The thick blue lines represent the self-similar solution of Day et al. (1998) and arrows indicate evolution due to an increase in time.

time. Both the first-stage pinch-off and the second-stage pinch-off show that $R_{\text {min }}$ follows $\left(t_{R}-t\right)^{2 / 3}$ before the occurrence of rupture, in agreement with a potential-flow scaling argument (e.g. Day et al. 1998; Lister \& Stone 1999). In the first-stage pinch-off the regime of $\left(t_{R}-t\right)^{2 / 3}$ starts around $R_{\min } \sim 0.4$, which is earlier than might be expected from an asymptotic analysis. In the second-stage pinch-off, several such regimes are observed, even very early on, when a minimum neck radius can first be identified. It is also interesting to see that the results for the first-stage pinchoff and the second-stage pinch-off are very close when the rupture is approached, e.g. $t_{R}-t<0.2$; in particular, both appear to transit to another regime almost at the same time just before pinch-off (as shown in the inset in figure 10). The effective interfacial thickness of the diffuse interface used in these simulations is 0.01 , which means that the pinching neck is well resolved at the times shown.

An approach to a self-similar shape of the pinching neck determined in Day et al. (1998), corresponding to the second $\left(t_{R}-t\right)^{2 / 3}$ regime in figure 10 , has been observed for second-stage pinch-off (see figure 9); however, it is at an early time for the first type of second-stage pinch-off that drop shapes (highlighted in red) approach the solution of Day et al. (1998) (see figure 9a), while the self-similar shapes appear to be approached only at a very late time for the second type, just before pinch-off. Unlike second-stage pinch-off, first-stage pinch-off was found not to exhibit self-similar surface shapes: the lower cone angle is found to be too low, as can be seen in figure 11(a). To investigate the origin of this, we show in figure $8(b)$ the location of the pinching (or almost pinching) neck in the $(r, z)$ plane for first-stage (along with second-stage and no) pinch-off. The pinching neck is seen to be very close to the solid substrate in first-stage pinch-off. This proximity of the solid substrate is expected to have a significant effect on the pinching neck, as the large downwards axial velocity in the neck (also associated with the self-similar solution Lister \& Stone 1999) is deflected by the substrate (this is also clearly seen in figure $12 a$, as discussed below). Further simulations have confirmed that this is a main cause of the difference (a separate difference is also discussed below): slow impact of a droplet into a pool of 


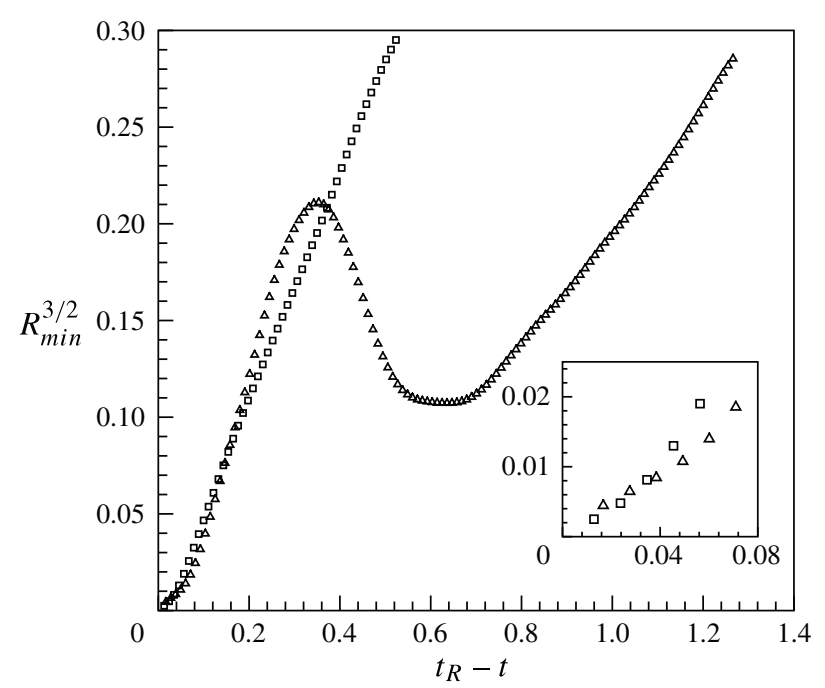

FIGURE 10. Comparison of numerical results with self-similar solutions at $W e=0.011$ and $O h=0.0066$. Pinching neck radius $R_{\min }$ versus time to rupture $t_{R}-t$ for first-stage pinchoff ( $\square$ ), with $\theta_{A}=15^{\circ}$ and $\Delta \theta=3^{\circ}$, and second-stage pinch-off $(\Delta)$, with $\theta_{A}=40^{\circ}$ and $\Delta \theta=10^{\circ}$. Here $t_{R}$ is the time of rupture.
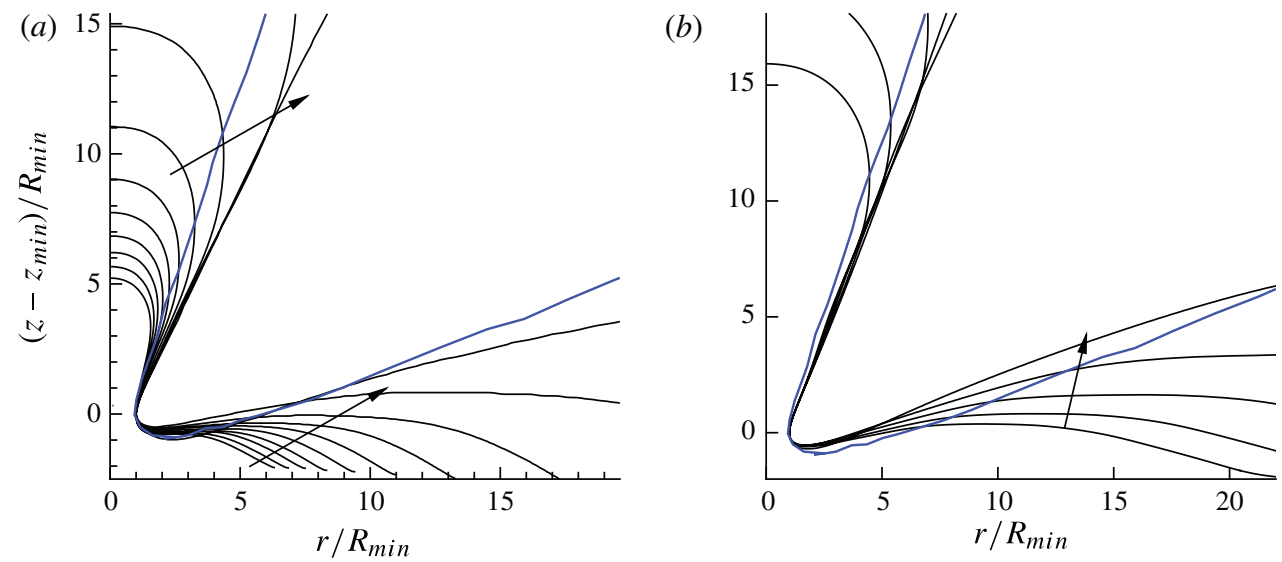

FIgURE 11. (Colour online) Comparison of scaled interface shapes for a spreading drop and a drop coalescing with a pool, at $W e=0.011$ and $O h=0.0066$ : $(a)$ scaled interfaces shape near the pinching neck for the same first-stage pinch-off case as in figure 10, corresponding to the last 10 data points in particular; $(b)$ the same drop as in $(a)$ but deposited onto a pool of the same liquid. The thick blue lines represent the self-similar solution of Day et al. (1998), arrows indicate evolution due to an increase in time.

the same liquid does show good agreement for the shape of the pinching neck region with Day et al. (1998) and clearly exhibits self-similar behaviour in an early $\left(t_{R}-t\right)^{2 / 3}$ regime, see figure $11(b)$.

For the second-stage pinch-off case in figure $9(a)$, figure $8(b)$ shows however that proximity of the substrate may not be the main cause of the deviation from the self-similar behaviour, as the pinching neck is much further removed from the wall. 
(a)

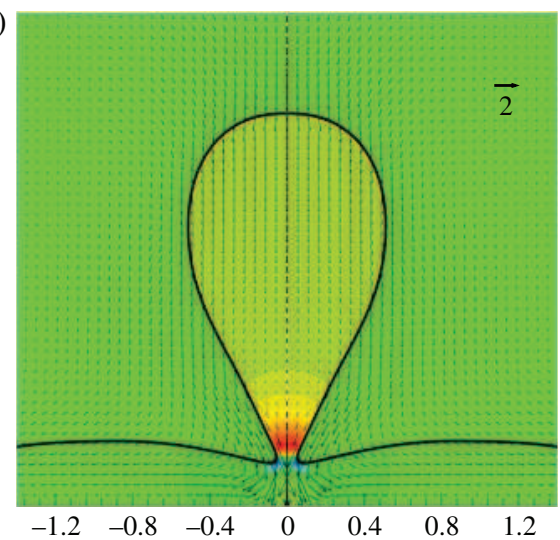

(b)

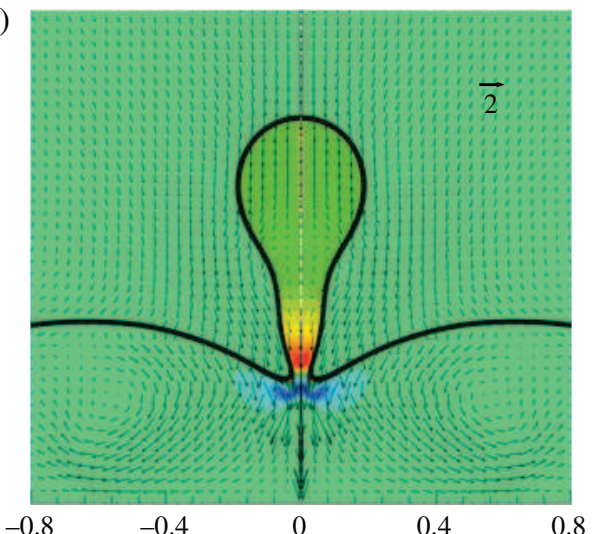

FIGURE 12. (Colour online) Evidence of interference of flow just below the pinching neck by the proximity of the solid substrate and by contact-line motion during the approach to first-stage pinch-off $(a) \theta_{A}=15^{\circ}$ and $\Delta \theta=3^{\circ}$ and second-stage pinch-off $(b) \theta_{A}=35^{\circ}$ and $\Delta \theta=10^{\circ}$. Both cases are at $O h=0.0066, W e=0.011$. The colour contours represent the pressure field, of which the value increases from blue to red. The vectors shown are the local velocity and their length is proportional to the magnitude of the velocity. A reference vector of 2 in magnitude is shown. The thick black line represents the interface and consists of the contours of volume fraction ranging from 0.1 to 0.9 .

The increasing proximity between the drop top and the pinching neck, which leads to the bulging-out of the upper cone and an increasing cone angle, is more likely to be relevant here. Evidently, it happens more or less at the same time as the deviation from self-similarity in figure $9(a)$. This is also supported by the notion that closer agreement with self-similar theory is reached in the second-stage pinch-off shown in figure $9(b)$, in which the length of the upper cone slightly increases with time. Inspection of our results shows that the rapid lowering of the drop height (figure 8a) is accompanied by a pressure built up underneath the very top of the droplet (which reaches a local maximum just above the pinching neck). A region of relatively low pressure between the neck and the top of the drop results in motion of liquid radially outwards and a local 'fattening' of the cone. This outwards motion can be seen to occur in both pinch-off regimes in figure 12, taken before pinch-off. The resulting fattening of the upper cone is especially apparent in figure $9(a)$.

A further complication can be identified in figure 12(b). In second-stage pinch-off it is seen that liquid is transported downwards only in a region around the symmetry axis and drawn radially inwards, to sustain the mass flux necessary for spreading. Part of the liquid is supplied by the large axial velocity inside the pinching neck, which is also present in the self-similar regime (cf. Day et al. 1998). However, the velocity vector plot in figure $12(b)$ shows that this is insufficient for the rapid spreading motion, as the surrounding region beneath the pinching neck is also drained towards the symmetry axis, which is not seen in first-stage pinch-off.

\subsection{Contact-line dynamics}

In all of the cases studied here, the spreading process is in an inertial-capillary regime (Biance et al. 2004): the contact-line radius $R_{c l}$ is seen in figure 13(a) to satisfy $R_{c l} \sim t^{1 / 2}$ for almost the entire duration of the spreading process, for both experiments and simulations. The time variation of apparent contact angle $\theta_{a p p}$ for the 

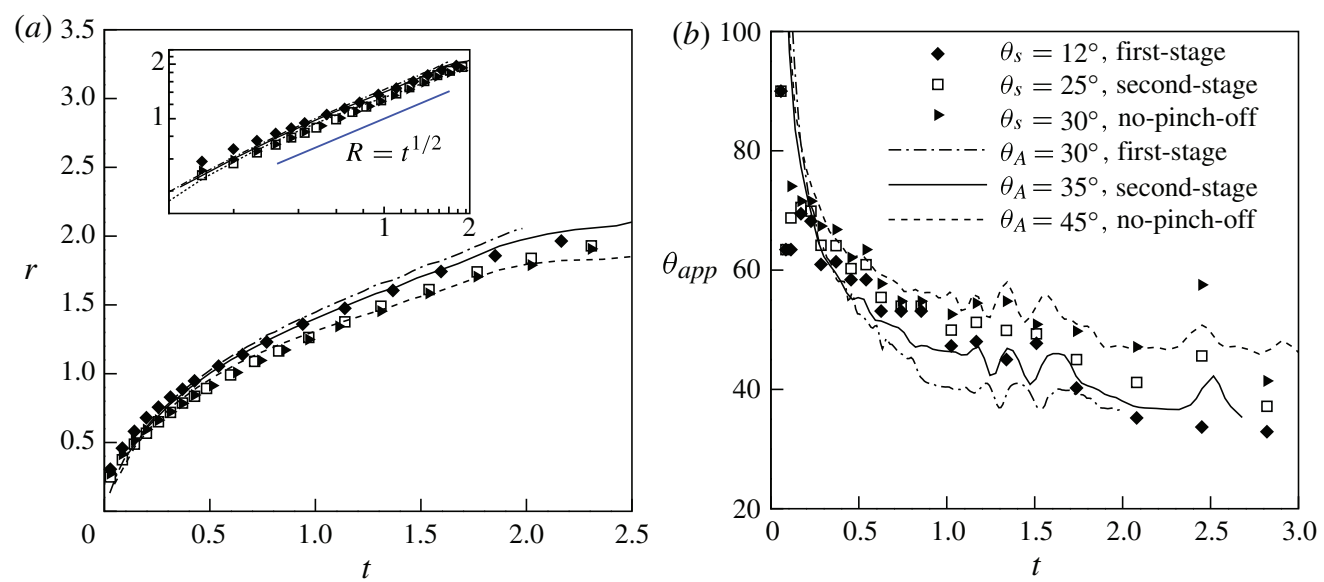

FIGURE 13. (Colour online) Fast spreading with different hydrophilicity, at $O h=0.0066$ and $W e=0.011$. (a) Radius of the wetted area and $(b) \theta_{a p p}$ as a function of time. Symbols and lines represent the experimental and numerical results, respectively.

three pinch-off regimes is shown in figure 13(b). In our simulations $\theta_{a p p}$ is defined as the maximum angle between the interface and the horizontal within a distance of 0.1 from the contact line (for a comparison against asymptotic theory for slow spreading using this definition, see Spelt (2005) and Ding \& Spelt (2007a)). The experimental and numerical results look similar, particularly the time of appearance and the pattern of oscillations in $\theta_{a p p}$ (also see figure 16 for experimental results with much better time resolution). A typical example of $\theta_{a p p}$ versus the dimensionless contact-line speed $C a_{c l}$ is shown in figure 14 for a no pinch-off case. Interestingly, $\theta_{a p p}$ appears not to be a single-valued function of $C a_{c l}$, consistent with previous observations of inertial effects in droplet spreading (Ding \& Spelt 2007a). In fact, the curve here is even more involved than the results in Ding \& Spelt (2007a) due to the stronger capillary waves encountered, with now several loops, which are seen to coincide with the collapse of the central part of the droplet (Ding \& Spelt 2007a), and the approach to a first-stage and second-stage pinch-offs.

Qualitatively similar behaviour is also observed in experiments, although the loops are more flattened there, as can be seen in figure 15. The loops in figure $15(b, c)$ are highly complex, but their extent is well beyond experimental uncertainty. The initial loop, figure $15(b)$, is seen to be mainly an oscillation in the capillary number along the same curve. However, the final loop exhibits a multivalued relation between contact angle and contact-line speed as in the simulations. The contact-line velocity is even seen to change sign there. Also shown is the prediction from Cox-Voinov-type theory, according to which (Hocking 1983)

$$
\theta_{a p p}^{3}=\theta_{A}^{3}+9 C a_{c l} \ln \left(\frac{R_{c l} \theta_{A}}{6 \mathrm{e} \lambda}\right),
$$

where $\lambda$ is the dimensional slip length; the contact angle $\theta_{A}$ is in radians (note, the dash-dotted curve in figure 15 has been obtained by estimating the total argument of the $\log$ term as $10^{5}$ for simplicity; for further refinement of the theory wherein the argument of the logarithm depends on the contact-line speed, see Eggers \& Stone (2004)). The value of $\lambda$ has been chosen here to correspond to a slip length of 

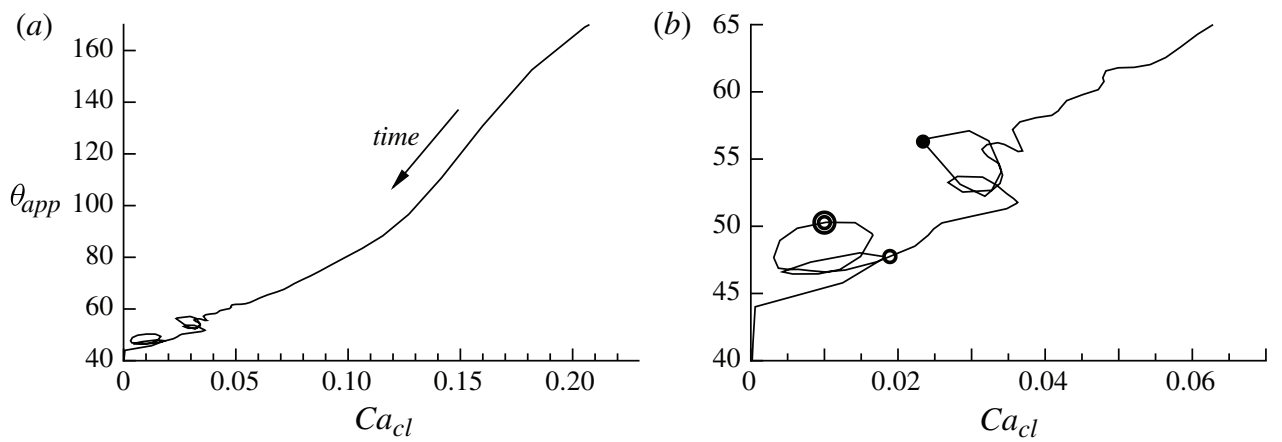

FIGURE 14. Numerical $\theta_{\text {app }}$ as a function of dimensionless instantaneous contact-line speed $C a_{c l}\left(=\mu U_{c l} / \sigma\right)$, for the case at $O h=0.0066, W e=0.011, \theta_{A}=45^{\circ}$ and $\Delta \theta=15^{\circ}$. This case lies in a no pinch-off regime. The right panel is a detail of the left panel. The solid circle indicates the instance where the initial drop reaches maximum height; the open and double circles indicate the points where nearly first- and second-stage pinch-off occurs, respectively.
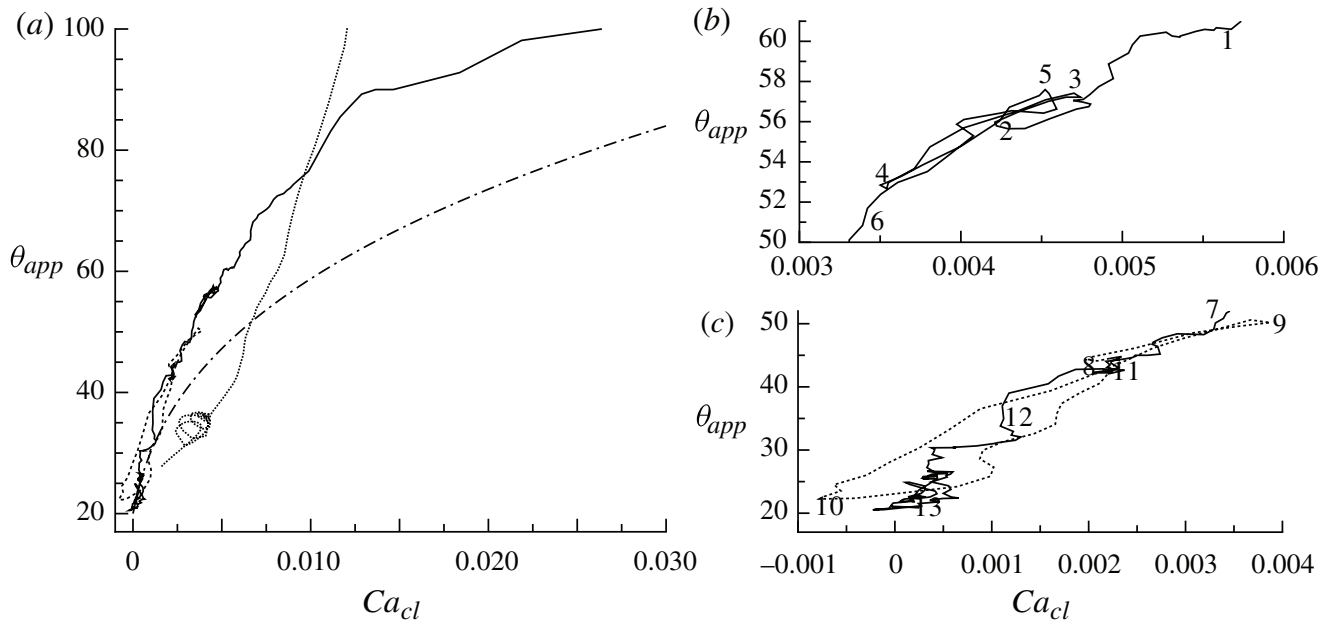

FIGURE 15. Experimental $\theta_{a p p}$ versus $C a_{c l}$. Second-stage pinch-off for a water drop of $0.77 \mathrm{~mm}$ in diameter with an impact speed of $0.079 \mathrm{~m} \mathrm{~s}^{-1}(O h=0.006$ and $W e=0.033)$ and $\theta_{s}=23^{\circ}$. The dashed line in $(a)$ and $(c)$ represents the main loop in the spreading from dimensionless $t=1.75-2.2$; more details are shown in figure 16(c). In $(a)$, the dash-dotted line represents the Cox-Voinov result for a slip length of approximately $1 \AA$, and the dotted line represents AMR results at $C n=0.001, O h=0.005$ and $\theta_{A}=18^{\circ}$. In (c) first-stage pinch-off nearly occurs at point 8 ; second-stage pinch-off takes place at the same point in this graph. The uncertainty in the experimental measurement is $\sim 1^{\circ}$ for $\theta_{a p p}$ and 0.0005 for $C a_{c l}$.

molecular size, $\lambda=1 \AA$, which has been found to work in, e.g., the experiments of Rio et al. (2005). It is seen that the theory under-predicts these results (and would have underestimated these even further if a slip length corresponding to the root-meansquare roughness height had been used, which is of $O(1 \mathrm{~nm})$ ); tests show that an unrealistically small slip-length value of $10^{-17} \mathrm{~m}$ would be needed for a reasonable agreement over the entire range of $C a_{c l}$ values shown. We revisit this issue in $\S 3.5$ below, where high-resolution simulations are discussed. 

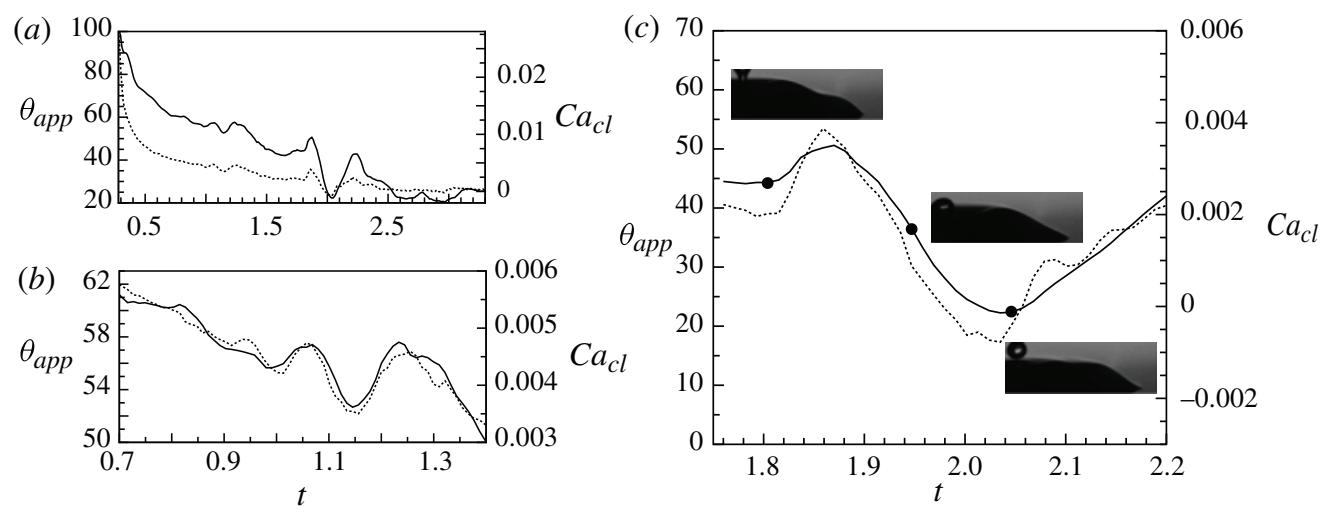

FIGURE 16. Detailed $\theta_{\text {app }}$ (solid lines) and $C a_{c l}$ (dashed lines) as a function of dimensionless time, from the experiment of figure 15. The images in $(c)$ are (from left to right) at the instants of the onset of second-stage pinch-off, the daughter droplet landing on the remainder of the mother drop and the daughter being launched into the air.

The results are more clear when plotted as time signals (figure 16). Two distinct oscillations are seen to occur (see also figure 13b). The first, shown in figure 16(b), occurs just after the top of the drop has started to come down, and vanishes at the point when first-stage pinch-off nearly takes place (at $t=1.5$; second-stage pinch-off occurs at $t=1.81$ ). This period coincides with the return of capillary waves spurred by the initial contact-line motion, after a 'reflection' at the symmetry axis. Coming with it, liquid is rapidly moved towards the contact-line region which induces inertial effects on the contact-line motion akin to those observed in Ding \& Spelt (2007a). Indeed, the oscillations in velocity are clearly seen to be very similar to those observed in those earlier simulations. The second oscillation, shown in figure 16(c), results from the waves generated by the expansion of the pinching neck at $t=1.5$ and appears to be much stronger in amplitude than the first, even leading to the recoil of the contact line. We further note here that the apparent contact angle and contact-line speed are seen to be in phase, suggesting the significant interaction of capillary waves with the contact-line motion. Close inspection of results shows the loops in figures 14(a) and 15(a) coincide with major capillary-type waves reaching the contact-line region. For example, the main loop indicated by the dashed line in figure 15 follows the neck expansion after the nearly first-stage pinch-off (see figure 16c).

\subsection{Pinch-off criteria and the role of the effective slip length}

The results presented in $\S 3.2$ show that, as in coalescence of non-equal-size drops (Zhang et al. 2009) and, for that matter, in prior studies for droplet impact into a pool, inertial effects must be sufficiently large for waves not to be damped, which suggests a critical value of $O h$ below which pinch-off could be expected. Also, the source of the wave is required to be strong. The wave source is determined largely by hydrophilicity in the present system, and by the ratio of drop sizes in coalescence of non-equal-size drops; we have found also that the contact line moves faster at lower values of $\mathrm{Oh}$ (Ding \& Spelt 2007a). Therefore, the flow regime map in figure 3 is in terms of $\mathrm{Oh}$ and the contact angle. Zhang et al. (2009) provided the pinch-off criteria of coalescence of non-equal-size drops (e.g. their figure $3 b$ ) by experiments. These criteria cannot apply directly to the first-stage pinch-off in the present system because the pinch-off process is significantly affected by the presence of the substrate 
and the contact-line motion. However, they may be, with some justification, applicable to the second-stage pinch-off, in which the influence of the substrate and the contactline motion become rather weak, if the expansion of the nearly pinched neck after the failed first-stage pinch-off is taken as the starting point of coalescence. This hypothesis is examined by checking the cases shown in the present figure 2(a,c), for which the $O h$ values of the upper 'droplet' (in terms of the system studied by Zhang et al. (2009)) are 0.007 and 0.01 , respectively, and the ratios of the lower to the upper 'droplet' are similar, 4-5. Therefore, from Zhang et al. (2009), we would expect 'first-stage pinch-off' in their terminology (i.e. second-stage pinch-off in the present system), rather than 'second-stage pinch-off' (i.e. third-stage pinch-off in the present system) or no pinch-off. This expectation is therefore in agreement with the experimental and the numerical results in this paper. Incidentally, we have not observed a third-stage pinch-off regime, neither in experiment nor in computation. Should such a regime exist, it would be expected at the boundary between the no pinch-off and second-stage pinch-off regimes in figure 3, which leaves very little room, which is the likely explanation why this has eluded observation.

A series of high-resolution (AMR) simulations has been performed at $O h=0.005$, $\theta_{s}=18^{\circ}$ for different values of Cahn number. We found no pinch-off to occur at $C n=0.55 / 640=0.00086$, first-stage pinch-off at $C n=1 / 640=0.0016$ and secondstage pinch-off at $C n=0.65 / 640=0.001$. From comparisons with a level-set method based on a slip formulation we estimate that this would correspond to changing an effective slip length from 80 to $150 \mathrm{~nm}$ for a millimetre-size drop. Successive drop shapes for the case wherein second-stage pinch-off is observed are shown at key stages in figure 17. These are found to agree with experimental images for approximately the same values of $O h$ and static angle (figure $2 a, b$ ) quite well. We therefore conclude that the length scale associated with the inner region near the contact line is of the order of $100 \mathrm{~nm}$ in the experiment. Although this is well above the size of roughness elements on the surfaces used in the present experiments, which is estimated to be around $1 \mathrm{~nm}$ (see $\S 2.1$ ), it is within the range of values inferred from experiments using theory in the case of slow spreading (Marsh et al. 1993). Either chemical inhomogeneities or a further interaction between the drop and surrounding air that is not modelled here (e.g. phase change or small dust particles near the contact line) may have led to this size of the contact-line region. The low value of the effective slip length in these simulations also make the correlation between $\theta_{a p p}$ and $C a_{c l}$ closer to the experimental results, regarding the range of $C a_{c l}$ for the loops to occur (compare, e.g. figures 14 and $15 a$ ). In future work, the effect of the definition of apparent contact angle used in simulations will be assessed.

\subsection{Coalescence cascade}

In most experiments the daughter droplet bounces off the surface of the mother drop. However, comprehensive experimental observations have revealed the occasional occurrence of a coalescence cascade, akin to that identified in Thoroddsen \& Takehara (2000) for a drop coalescing with a flat liquid surface. Figure 18 shows a realization with a sequence of six first-stage pinch-offs (see supplementary movie 2). This case corresponds to very similar dimensionless groups as the second-stage pinch-off in figure 15, but with a more hydrophilic substrate. The diameter ratios of daughter to mother droplets are $0.45,0.63,0.56,0.60,0.58$ and 0.59 , which are somewhat larger than the value 0.48 observed in droplet ejection in coalescence of non-equal-size drops (see Zhang et al. 2009), although the present cascade is affected by the rapid vertical motions of the lower free surface in the latter case. The smallest daughter droplet 
(a)

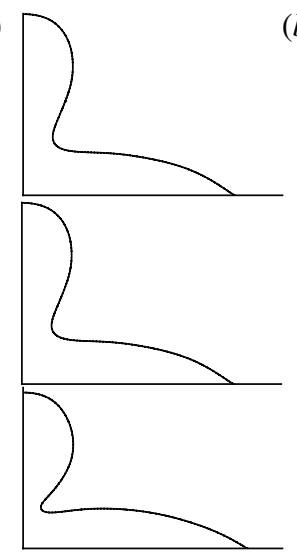

(b)

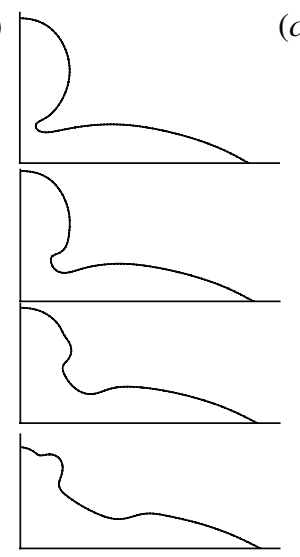

$(c)$

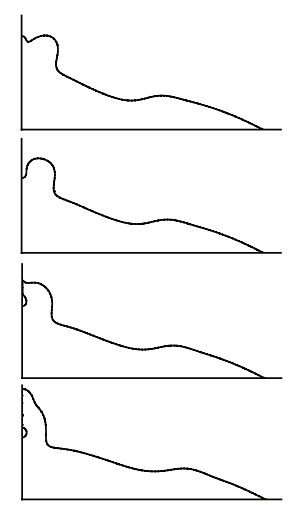

(d)

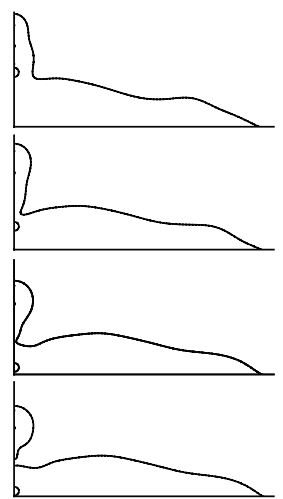

FIGURE 17. Selected snapshots of the numerical simulation results with AMR showing various stages of the process: $(a)$ approach to first-stage pinch-off, $(b)$ re-expansion of the neck, $(c)$ impact of secondary capillary wave on top of droplet thereby entraining a small bubble and $(d)$ second-stage pinch-off.

in the last panel of figure 18 is $26 \mu \mathrm{m}$ in diameter, which is in excellent agreement with the prediction by Blanchette \& Bigioni (2006), who predict for water a smallest possible daughter diameter of $22 \mu \mathrm{m}$. It is interesting to note that the coalescence of two bubbles produces a much smaller satellite bubble where this daughter/mother ratio is around 0.1, see Zhang \& Thoroddsen (2008).

The second stage in this cascade differs from second-stage pinch-off. Evidently, the impact of the first-generation ejected droplet and subsequent coalescence with the main droplet creates a disturbance on the top of the latter, which reaches the contact-line region, returns to the top of the centre of the drop and finally leads to what is again similar to a first-stage pinch-off, after which this process repeats itself. In second-stage pinch-off, as can be seen most clearly in figure 2(c), after first-stage pinch-off has failed, the re-expanding neck creates a new wave that propagates further upwards; the remainder of the droplet is only to a limited extent involved in this wave propagation, unlike in the cascade.

A pinch-off event after first-stage pinch-off and coalescence of the daughter and mother drops has also been observed in numerical simulations when using AMR. This was for $O h=0.005, \theta_{s}=18^{\circ}$ and $\epsilon=\Delta x$. In this simulation, a relatively large bubble was entrapped upon the coalescence of the first daughter droplet with the mother drop and that the second pinch-off occurred just above this bubble. We would expect this bubble to have an effect on the pinch-off process, although it is of course of interest to note here that such a second pinch-off has been observed in a simulation (given the presence of this bubble, this expensive simulation has not been continued to establish whether subsequent pinch-off events occurred). Bubble entrapment has been observed experimentally during coalescence of a pinched droplet and a larger drop (e.g. Thoroddsen, Etoh \& Takehara 2003, 2007a). The underlying mechanism can be understood from the pressure build-up in the narrow gap between the two drops as the gas tries to escape but is slowed down by shear stress at the surfaces of the drop, since the pressure build-up causes deformation of the droplets (e.g. Yiantsios \& Davis 1990). We note here that in numerical methods such as that utilized here, coalescence is expected to occur when the distance between two approaching interfaces becomes of the order of the thickness of the interface (i.e. the grid spacing), which normally is 


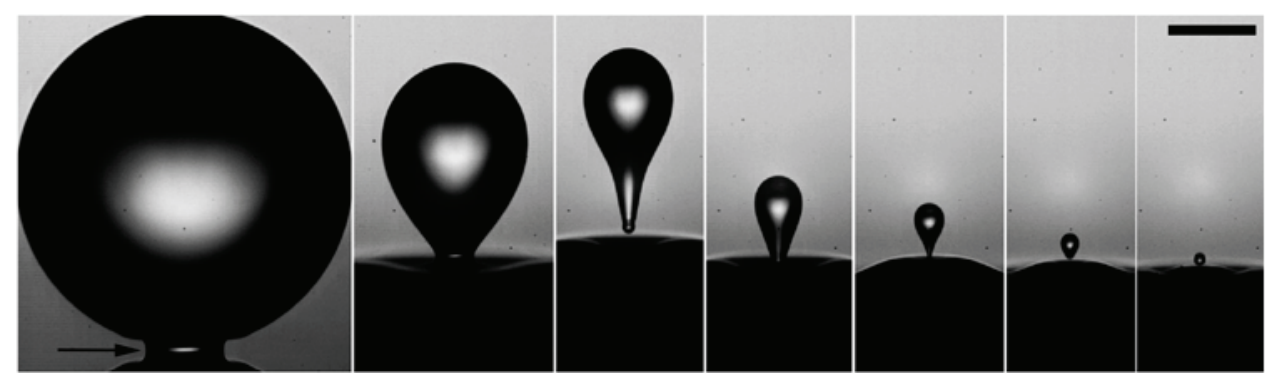

FIGURE 18. Six-stage coalescence cascade after first-stage pinch-off for a water drop of $0.77 \mathrm{~mm}$ in diameter, with an impact speed of $0.079 \mathrm{~m} \mathrm{~s}^{-1}(\mathrm{Oh}=0.006, W e=0.033)$ and $\theta_{s}=12 \pm 2^{\circ}$. Snapshots are shown at dimensionless $t=0.027,1.72,3.35,6.53,7.0,7.28$ and 7.39 from the start of spreading. The arrow points at the location of the glass plate and the scale bar is $200 \mu \mathrm{m}$ long. A full video accompanies this paper (supplementary movie 2).

before the point when long-range intermolecular forces are expected to play a role in experiments. Hence, coalescence occurs earlier than in experiment, i.e. when the gap between the two drops is still somewhat larger than in experiment, and a relatively large bubble is entrapped.

\section{Conclusions}

We have demonstrated a new regime of droplet ejection during the slow deposition of drops on a wall, in cases of complete or near-complete wetting. The regime produces daughter droplets that are at most half in radius compared with those resulting from first-stage pinch-off. Such a regime has only been found in experiments on the coalescence of unequal-sized drops (Zhang et al. 2009). The present work confirms the relevance of such mechanism of breakup to a broader range of systems. Also, numerical results wherein this regime has been successfully simulated are reported here for the first time; high-resolution numerical simulations show quantitative agreement with experiments when allowing for an effective slip length in the formulation of the numerical method as a fitting parameter.

The ejection phenomena originate from the focusing of a wave that starts at the contact line. The numerical results have shown that this wave propagation is closely related to the self-similar behaviour away from the contact line, similar to inviscid recoil of droplets (Keller \& Miksis 1983; Billingham 1999), and that the phase velocities of capillary waves do not depend on $\theta_{A}$ and $O h$. This is all despite the different geometry involved in recoil and in the present problem. The simulations also reveal that good agreement with the self-similar solution of Day et al. (1998) is obtained when the droplet merges with a liquid layer for almost the entire pinch-off process. The case of a droplet impacting a dry wall differs crucially in this respect: although the pinching neck radius decreases as a function of time as predicted by a scaling argument, the double-cone shape of the pinching region does differ from the self-similar solution of Day et al. (1998), in both ejection mechanisms. The results indicate that the deviation from the geometric self-similarity is due to the interference in the neck region by the proximity of the wall, a downwards acceleration of the top of the drop and the rapid drop spreading. The radially inwards motion of the pinching neck appears to be insufficient in either case to enforce an eventual approach to the self-similar potential flow solution in these flows. The present work therefore adds evidence of the limitations of self-similar theory that is of a different nature to 
that reported in Doshi et al. (2003). The experiments have also revealed that beyond droplet pinch-off, a coalescence cascade (Thoroddsen \& Takehara 2000) can occur.

Detailed experimental results for an apparent contact angle and contact-line speed have also been presented, confirming for the first time the qualitative behaviour identified previously in numerical simulations (Ding \& Spelt 2007a), which has also been seen recently in numerical simulations of reactive wetting (Wheeler, Warren \& Boettinger 2010). A remaining difference between the experimentally measured apparent contact angle and an apparent angle inferred from simulations (in spite of the good agreement of the overall flow behaviour), along with detailed comparisons with theoretical analyses, is investigated further in future work.

\section{Acknowledgements}

H.D. was supported by 100 Talents Program of The Chinese Academy of Sciences and the National Natural Science Foundation of China (Grant No. 11172294). E.Q.L. and S.T.T. were partly supported by KAUST-BERKELEY AEA grant 7000000024. Y.S. and P.D.M.S. were supported from EPSRC under grant numbers EP/E046029/1.

Supplementary movies are available at http://dx.doi.org/10.1017/jfm.2012.49.

\section{REFERENCES}

Biance, A.-L., Clanet, C. \& Queré, D. 2004 First steps in the spreading of a liquid droplet. Phys. Rev. E 69, 016301.

BillinghaM, J. 1999 Surface-tension-driven flow in fat fluid wedges and cones. J. Fluid Mech. 397, $45-71$.

Blanchette, F. \& Bigioni, T. P. 2006 Partial coalescence of drops at liquid interfaces. Nature Phys. 2, 254-257.

Blanchette, F. \& Bigioni, T. P. 2009 Dynamics of drop coalescence at fluid interfaces. J. Fluid Mech. 620, 333-352.

Cox, R. G. 1986 The dynamics of the spreading of liquids on a solid surface. Part 1. Viscous flow. J. Fluid Mech. 168, 169.

DAY, R. F., Hinch, E. J. \& LiSTER, J. R. 1998 Self-similar capillary pinch off of an inviscid fluid. Phys. Rev. Lett. 80, 704-707.

Ding, H. \& SPELT, P. D. M. $2007 a$ Inertial effects in droplet spreading: a comparison between diffuse interface and level-set simulations. J. Fluid Mech. 576, 287-296.

Ding, H. \& SPELT, P. D. M. $2007 b$ Wetting condition in diffuse interface simulation of contact line motion. Phys. Rev. E 75, 046708.

Ding, H., Spelt, P. D. M. \& Shu, C. 2007 Diffuse interface model for incompressible two-phase flows with large density ratios. J. Comput. Phys. 226, 2078-2095.

Ding, H. \& Spelt, P. D. M. 2008 Onset of motion of a 3D droplet on a wall in shear flow at moderate Reynolds numbers. J. Fluid Mech. 599, 341-362.

Ding, H., Gilani, M. N. H. \& Spelt, P. D. M. 2010 Sliding, pinch off and detachment of a droplet on a wall in shear flow. J. Fluid Mech. 644, 217-244.

Doshi, P., Cohen, I., Zhang, W. W., Siegel, M., Howell, P., Basaran, O. A. \& Nagel, S. R. 2003 Persistence of memory in drop breakup: the breakdown of universality. Science 302, $1185-1188$.

EgGers, J. 1997 Nonlinear dynamics and breakup of free-surface flows. Rev. Mod. Phys. 69, 865-929.

EgGers, J. \& Stone, H. A. 2004 Characteristic lengths at moving contact lines for a perfectly wetting fluid: the influence of speed on the dynamic contact angle. J. Fluid Mech. 505, 309-321.

DE Gennes, P. G. 1985 Wetting: Statistics and dynamics. Rev. Mod. Phys. 57, 827-863. 
Gilet, T., Mulleners, K., Lecomte, J. P., Vandewalle, N. \& Dorbolo, S. 2007 Critical parameters for the partial coalescence of a droplet. Phys. Rev. E 75, 036303.

Goriely, A. \& McMillen, T. 2002 Shape of a cracking whip. Phys. Rev. Lett. 88, 244301.

Hocking, L. M. 1983 The spreading of a thin drop by gravity and capillarity. Q. J. Mech. Appl. Maths 36, 55-69.

JACQMIN, D. 1999 Calculation of two-phase Navier-Stokes flows using phase-field modelling. J. Comput. Phys. 155, 96-127.

JACQMin, D. 2000 Contact-line dynamics of a diffuse fluid interface. J. Fluid Mech. 402, 57.

JANG, E. Y., SEO, D. K., KIM, T., KANG, T. J. \& KIM, Y. H. 2010 Electrical resistance variation of carbon-nanotube networks due to surface modification of glass substrate. Phys. Status Solidi A 1.

Keller, J. B. \& Miksis, M. J. 1983 Surface tension driven flows. SIAM J. Appl. Maths 43, 268-277.

KING, A. C. 1991 Moving contact lines in slender fuid wedges. Q. J. Mech. Appl. Maths 44, 173-192.

Lawrie, J. B. 1990 Surface-tension-driven flow in a wedge. Q. J. Mech. Appl. Maths 43, 251-273.

Lister, J. R. \& Stone, H. A. 1999 Capillary breakup of a viscous thread surrounded by another viscous fluid. Phys. Fluids 10, 2758-2764.

Macneice, P., Olson, K. M., Mobarry, C., DeFainchtein, R. \& Packer, C. 2000 PARAMESH: a parallel adaptive mesh refinement community toolkit. Comput. Phys. Commun. 126, 330-354.

Manasseh, R., Riboux, G. \& Risso, F. 2008 Sound generation on bubble coalescence following detachment. Intl J. Multiphase Flow 34, 938-949.

Marsh, J. A., Garoff, S. \& Dussan, V. E. B. 1993 Dynamic contact angles and hydrodynamics near a moving contact line. Phys. Rev. Lett. 70, 2778-2781.

Rayleigh, L. 1879 On the capillary phenomena of jets. Proc. R. Soc. 29, 71-97.

Renardy, Y., Popinet, S., Duchemin, L., Renardy, M., Zaleski, S., Josserand, C., Drumright-Clarke, M. A., Richard, D., Clanet, C. \& Quéré, 2003 Pyramidal and toroidal water drops after impact on a solid surface. J. Fluid Mech. 484, 69-83.

Rio, E., Daerr, A., Andreotti, B. \& Limat, L. 2005 Boundary conditions in the vicinity of a dynamic contact line: experimental investigation of viscous drops sliding down an inclined plane. Phys. Rev. Lett. 94, 024503.

Rioboo, R., Adao, M. H., Voué, M. \& De Coninck, J. 2006 Experimental evidence of liquid drop breakup in complete wetting experiments. J. Mater. Sci. 41, 5068-5080.

Roux, D. C. D. \& Cooper-White, J. J. 2004 Dynamics of water spreading on a glass surface. J. Colloid Interface Sci. 277, 424-436.

Sierou, A. \& Lister, J. R. 2004 Self-similar recoil of inviscid drops. Phys. Fluids 16, $1379-1394$.

Shaw, S. J. \& Spelt, P. D. M. 2010 Shock emission from collapsing gas bubbles. J. Fluid Mech. 646, 363-373.

SPELT, P. D. M. 2005 A level-set approach for simulations of flows with multiple moving contact lines with hysteresis. J. Comput. Phys. 207, 389-404.

TANner, L. H. 1979 The spreading of silicone oil drops on horizontal surfaces. J. Phys. D 12, $1473-1483$.

Thoroddsen, S. T., Etoh, T. G. \& Takehara, K. 2003 Air entrapment under an impacting drop. J. Fluid Mech. 478, 125-134.

Thoroddsen, S. T., Etoh, T. G. \& TAkehara, K. $2007 a$ Microjetting from wave focusing on oscillating drops. Phys. Fluids 19, 152101.

Thoroddsen, S. T., Qian, B., ETOH, T. G. \& TAKehara, K. $2007 b$ The initial coalescence of miscible drops. Phys. Fluids 19, 072110.

Thoroddsen, S. T. \& Takehara, K. 2000 The coalescence cascade of a drop. Phys. Fluids 12, $1265-1267$.

Wheeler, D., Warren, J. A. \& Boettinger, W. J. 2010 Modelling the early stages of reactive wetting. Phys. Rev. E 82, 051601. 


\section{H. Ding, E. Q. Li, F. H. Zhang, Y. Sui, P. D. M. Spelt and S. T. Thoroddsen}

YARIN, A. L. 2006 Droplet impact dynamics: splashing, spreading, receding, bouncing. Annu. Rev. Fluid Mech. 38, 159-192.

Yiantsios, S. G. \& DaVis, R. H. 1990 On the buoyancy-driven motion of a drop towards a rigid or a deformable surface. J. Fluid Mech. 217, 547-573.

Zhang, F. H. \& Thoroddsen, S. T. 2008 Satellite generation during bubble coalescence. Phys. Fluids 20, 022104.

Zhang, F. H., Li, E. Q. \& Thoroddsen, S. T. 2009 Satellite formation during coalescence of unequal size drops. Phys. Rev. Lett. 102, 104502. 